OPEN ACCESS

Edited by:

Marc Pera-Titus,

Fudan University, China

Reviewed by:

Yusuke Yamauchi,

The University of

Queensland, Australia

Taner Yonar

Uludag University, Turkey

*Correspondence:

Tao Yang

tao.yang@hhu.edu.cn

Xingtao Xu

xingtao.xu@hhu.edu.cn

Specialty section:

This article was submitted to Green and Sustainable Chemistry, a section of the journal

Frontiers in Chemistry

Received: 23 June 2020 Accepted: 29 October 2020 Published: 30 November 2020

Citation:

Lin P, Liao M, Yang T, Sheng X, Wu Y and $X u X$ (2020) Modification of

Metal-Organic Framework-Derived Nanocarbons for Enhanced Capacitive

Deionization Performance: A

Mini-Review. Front. Chem. 8:575350 doi: $10.3389 /$ fchem.2020.575350

\section{Modification of Metal-Organic Framework-Derived Nanocarbons for Enhanced Capacitive Deionization Performance: A Mini-Review}

\author{
Peng Lin ${ }^{1}$, Maoxin Liao ${ }^{1}$, Tao Yang ${ }^{1,2 *}$, Xinran Sheng ${ }^{1}$, Yue $W u^{1}$ and Xingtao $X u^{1 *}$ \\ ${ }^{1}$ College of Hydrology and Water Resources, Hohai University, Nanjing, China, ${ }^{2}$ State Key Laboratory of Hydrology-Water \\ Resources and Hydraulic Engineering, Hohai University, Nanjing, China
}

Capacitive deionization $(\mathrm{CDI})$ is a promising electrochemical water treatment technology. Development of new electrode materials with higher performance is key to improve the desalination efficiency of CDI. Carbon nanomaterials derived from metal-organic frameworks (MOFs) have attracted wide attention for their porous nanostructures and large specific surface areas. The desalination capacity and cycling stability of MOF-derived carbons (MOFCs) have been greatly improved by means of morphology control, heteroatom doping, Faradaic material modification, etc. Despite progress has been made to improve their CDI performance, quite a lot of MOFCs are too costly to be applied in a large scale. It remains crucial to develop MOFCs with both high desalination efficiency and low cost. In this review, we summarized three modification methods of MOFCs, namely morphology control, heteroatom doping, and Faradaic material doping, and put forward some constructive advice on how to enhance the desalination performance of MOFCs effectively at a low cost. We hope that more efforts could be devoted to the industrialization of MOFCs for CDI.

Keywords: capacitive deionization, nanocarbon, metal-organic framework, modification, desalination

\section{INTRODUCTION}

With the increasing shortage of water resources worldwide, the exploration of new methods for water treatment has become one of the important ways to solve the problem (Xu et al., 2017b; Sun et al., 2020a,b). Capacitive deionization (CDI) is considered a promising water treatment technology with powerful competitiveness compared with reverse osmosis and electroosmosis owing to its advantages of low energy consumption, environmental friendliness, and low cost (Oren, 2008). It shows excellent performance in the fields of seawater desalination, brackish water desalination, heavy metal ion removal (Hou et al., 2018), and element enrichment. So far, numerous materials (especially carbon materials) have been developed for CDI electrodes, including activated carbon (Wang et al., 2013; Luo et al., 2019), activated carbon nanofiber (ACF) (Wang et al., 2012), carbon aerogel (CA) (Jung et al., 2007), carbon nanotubes (CNT) (Wang et al., 2011), graphene (Xu et al., 2016b; Huang et al., 2019), ordered mesoporous carbons (OMCs) (Duan et al., 2015; Xu et al., 2019c), etc. Among them, graphene is undoubtedly the most promisingly studied electrode material for CDI mainly owing to its large specific area, low cost, and abundance (Li et al., 2012). However, its poor salt adsorption capacity (SAC) limits its further application. The development of CDI needs, first and foremost, low-cost and high-efficiency electrodes (AlMarzooqi et al., 2014). 
Carbon nanomaterials derived from metal-organic frameworks (MOFs) have attracted wide attention recently (Chaikittisilp et al., 2013; Xu et al., 2017a). Thanks to the porous structures and tailored compositions of precursors (Yaghi and Li, 1995; Wang, Z., et al., 2019), MOF-derived carbons (MOFCs) show adjustable pore structures, large specific surface areas, and good conductivity, giving them unparalleled CDI performance. Since Yang et al. demonstrated that carbon derived from IRMOF1 has the potential as a high-performance CDI electrode material (Yang et al., 2014), more and more MOFs have been used for producing CDI electrodes, including the well-known zeolitic imidazolate frameworks (ZIFs) (Liu et al., 2015b; Wang et al., 2017; Gao et al., 2018), Materials Institute Lavoisier (MILs) (Xu et al., 2016a; Wang, K., et al., 2019), and MOF-5 (Chang et al., 2015). Modifications, such as morphology control, heteroatom doping (Wang et al., 2014; Xu et al., 2015), and Faradaic material doping, have been further studied to construct nanomaterials with more reasonable structures and compositions. As a result, the SAC and cycling stability of MOFCs have been greatly improved. Nevertheless, a considerable portion of MOFCs are costly due to their complex synthesis and expensive precursors, which limits their application in a large scale. The efficient and low-cost modification of MOFCs still needs to be systematically explored.

In this paper, the principle of CDI is given, including its adsorption mechanism and requirements for electrode materials. Thereafter, three common modification methods in the aspects of morphology control by template, element doping, and Faradaic material doping are summarized (Figure 1). Moreover, we put forward some advice on cost control and discuss the future development direction of MOFCs for the desalination industry.

\section{THE PRINCIPLE OF CDI}

A typical CDI cell consists of two electrodes placed in parallel and saline water between them. The electrodes adsorb ions from saline water when charged and release ions when discharged, so as to desalinate feed water or recycle electrodes. The electrodes can be categorized into non-Faradaic electrodes and Faradaic electrodes according to the ion adsorption mechanism (Chen et al., 2020; Lu et al., 2020). In most carbon-based CDI processes, ions are usually stored in the electric double layers (EDLs) formed within the pores of porous electrodes without the occurrence of Faradaic reactions. For efficient and rapid desalination, electrode materials therefore should meet at least the following properties: (1) large specific surface area for ion storage and suitable pore structure for rapid migration of ions, (2) high conductivity for rapid transfer of electrons within the electrodes, (3) stable electrochemical property for cycling stability, and (4) good hydrophilicity (Yin et al., 2013; Liu et al., 2015a, 2017; Tang et al., 2019). To achieve these aims, morphology control and heteroatom doping have been frequently used. Aside from the commonly used non-Faradaic electrodes, Faradaic electrodes are also utilized to store ions mainly based on Faradaic reaction, which have attracted wide attention for their typical high SAC and cycling stability (Ding, Z., et al., 2019).

\section{MODIFICATION OF MOFCS FOR ENHANCED PERFORMANCE}

\section{Morphology Control With Templates}

Although MOFCs have high specific surface areas and high porosities, most MOF crystals are dissociative and solid particles, which can lead to poor electrical conductivity and low accessible surface area (Tang et al., 2016; Xu et al., 2020a). Morphology control with templates, including MOF templates and external templates (e.g., carbon materials, metal compounds, polymers), may be an effective method to optimize the nanostructures and composition of MOFCs (Dang et al., 2017; Xu et al., 2019b). ZIF-8 is a typical subfamily of MOFs that has been widely investigated for CDI application. Liu et al. prepared porous carbon polyhedrons (PCPs) through direct carbonization of ZIF-8, which showed an improved desalination performance (with a SAC of $13.86 \mathrm{mg} \mathrm{g}^{-1}$ ) and stability compared with commercial AC (Liu et al., 2015b). Subsequently, Xu et al. reported hierarchical porous carbon nanotubes (CNTs)/PCP hybrid (hCNTs/PCP) fabricated via in situ insertion of CNTs in ZIF-8 with a subsequent pyrolysis process. Thanks to its novel CNT-inserted-PCP porous structure, high specific surface area, and good electrical conductivity, the resultant hCNTs/PCP exhibited a high SAC of $20.5 \mathrm{mg} \mathrm{g}^{-1}$ and stable cycling stability (Xu et al., 2016d). After that, Xu et al. synthesized integrated MOF tubes by controlled growth of ZIF-8 nanocrystals on 3D polymeric fibers with the subsequent dissolution of template (Supplementary Figure 1). Afterwards, self-standing nitrogendoped carbon tubes (NCTs) with an ultrahigh SAC of $56.9 \mathrm{mg}$ $\mathrm{g}^{-1}$ were obtained by thermal conversion of the resulting MOF tubes (Xu et al., 2020b). The external templates can tune the morphology of MOFCs effectively; however, their market price might not be acceptable for practical application and sometimes require complicated template removal operation (Dang et al., 2017). More versatile and cheaper templates that effectively controlled the morphology are needed (Dutta et al., 2016; Xu et al., 2016c).

\section{Heteroatom Doping}

Heteroatom doping is a common modification method for improving the electrochemical performance of carbon materials (Li et al., 2018). Non-metallic elements or metal ions can be evenly doped in MOFCs by simple carbonization of MOF precursors containing target elements, which would contribute to enhancing the comprehensive properties of carbon materials including conductivity, hydrophilicity, and stability (Kurak and Anderson, 2009; Zheng et al., 2011; Cheng et al., 2019; Xu et al., 2019a). Gao et al. synthesized nitrogen-doped graphitic carbon polyhedrons (NGCPs) by direct carbonization of ZIF-8. NGCPs show a maximum SAC of $17.73 \mathrm{mg} \mathrm{g}^{-1}$ and high salt adsorption rate of $4.14 \mathrm{mg} \mathrm{g}^{-1} \mathrm{~min}^{-1}$ and good regeneration performance (Gao et al., 2018). Zhang et al. prepared $\mathrm{N}$, $\mathrm{P}$, S co-doped hollow carbon polyhedron (denoted as ZIF8@PZS-C) derived from ZIF-8-based core-shell nanocomposites (denoted as ZIF-8@PZS). The resultant ZIF-8@PZS-C displayed an improved electrical conductivity, excellent hydrophilic, and high SAC of $22.19 \mathrm{mg} \mathrm{g}^{-1}$ (Zhang et al., 2018). Considering 


\title{
Morphology Control
}

MOFs, Carbon Materials,

Metal Compounds,

Polymers

\section{Heteroatom Doping}

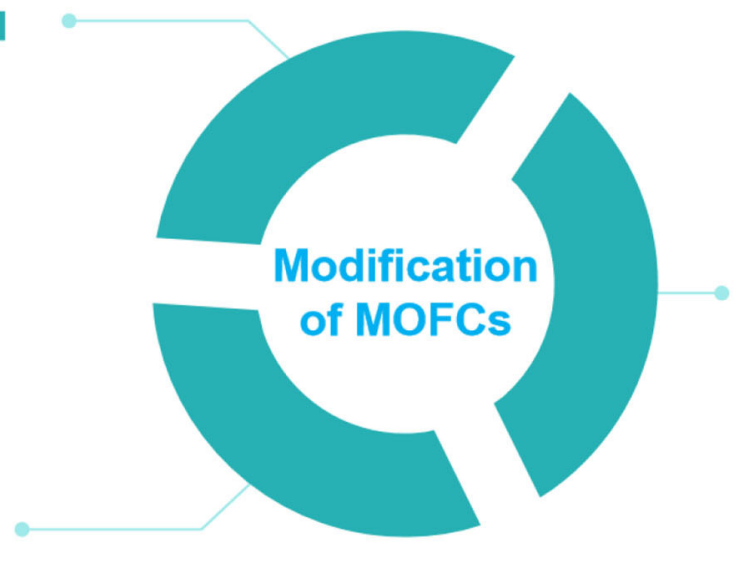

\section{Faradaic Material Doping}

Transition Metal Oxides,

Prussian Blue Analogs,

Polyanionic Phosphates,

Conducting Polymers,

Transition Metal

Dichalcogenides

\author{
Nitrogen, Phosphorus, \\ Sulfur, Chlorine, Iron
}

FIGURE 1 | Overview of modification methods of metal-organic framework (MOF)-derived carbons.

the performance fading of conventional carbon materials caused by the formation of $\mathrm{H}_{2} \mathrm{O}_{2}$ due to the reduction of dissolved oxygen in nature saline water, the introduction of oxygen reduction mechanism will effectively improve the stability of MOFCs (Luo et al., 2019). Xu et al. prepared nitrogen-irondoped carbon tubes (3D-FeNC tubes) derived from the 3D interconnected MOF tubes (Supplementary Figure 2). Thanks to its well-defined structure and enhanced oxygen reduction ability, the 3D-FeNC tubes achieved both excellent salt removal ability and cycling performance in oxygenated saline water (Xu et al., 2020a). The research reveals that high-performance oxygen reduction catalysts, such as $\mathrm{Fe}, \mathrm{N}$, and other heteroatom-doped carbon materials (Zhang et al., 2020), can significantly improve the continuous desalination performance of CDI. Heteroatom doping enables MOFCs with higher desalination capacity, faster adsorption rate, and more importantly, better stability. Dissolved oxygen ubiquitous in natural water will eventually cause the performance fading of carbon materials. By simply doping, the stability of MOFCs can be greatly improved, which contributes to their practical application for the desalination industry.

\section{Faradaic Material Doping}

Even though great progress has been made in improving the CDI performance of MOFCs based on EDLs, further improvement of SAC seems hard to achieve due to the limitation of physical charge adsorption capacity (Suss et al., 2015; Zhao et al., 2019). Inspired by the booming field of energy storage such as sodium-ion battery and supercapacitor (Liu et al., 2020). Faradaic materials have been investigated for CDI and proved to be promising candidates with high SAC and cycling stability (Tang, W., et al., 2019). Widely studied Faradaic materials include transition metal oxides (e.g., $\mathrm{MnO}_{2}, \mathrm{TiO}_{2}$, $\left.\mathrm{Na}_{4} \mathrm{Ti}_{9} \mathrm{O}_{20}\right)$, Prussian blue analogs, polyanionic phosphates [e.g., $\mathrm{FePO}_{4}, \mathrm{NaTi}_{2}\left(\mathrm{PO}_{4}\right)_{3}, \mathrm{Na}_{3} \mathrm{~V}_{2}\left(\mathrm{PO}_{4}\right)_{3}$ ], conducting polymers (e.g., polypyrrole, polyaniline), MXenes, transition metal dichalcogenides, and so on (Qin et al., 2019, 2020; Yu et al., 2019). Yang et al. prepared hierarchically porous carbon-coated zirconium oxide nanocubes (HCZ) derived from metal-organic framework (Zr-UiO-66) for CDI electrodes. The asymmetrical cell composed of $\mathrm{HCZ}$ negative electrode and AC positive electrode showed a remarkable SAC of $55.17 \mathrm{mg} \mathrm{g}^{-1}$ in $250 \mathrm{mg}$ $\mathrm{L}^{-1}$ aqueous sodium chloride solution at $1.4 \mathrm{~V}$ (Yang and Luo, 2019). Ding et al. reported a titanium dioxide/porous carbon composite $\left(\mathrm{TiO}_{2} @ \mathrm{PC}\right)$ derived from MIL-125 (Ti) for a membrane CDI. A synergy of high pseudocapacitance and good oxidation resistance endows the anatase $\mathrm{TiO}_{2} @ \mathrm{PC}$ (annealed at $600^{\circ} \mathrm{C}$ ) with an improved SAC of $46.7 \mathrm{mg} \mathrm{g}^{-1}$ at $10 \mathrm{~mA} \mathrm{~g}^{-1}$ and stable cycling performance over 50 cycles (Ding, M., et al., 2019). Wang et al. prepared MIL-125 (Ti)derived $\mathrm{NaTi}_{2}\left(\mathrm{PO}_{4}\right)_{3}$ /carbon (NTP/C) composite as electrode materials for hybrid CDI (HCDI; Supplementary Figure 3). Due to the unique porous structure, high specific surface area, and good electrical conductivity of NTP/C, the HCDI system with NTP/C composite cathode and AC anode exhibited an excellent desalination performance with a high SAC of $167.4 \mathrm{mg}$ $\mathrm{g}^{-1}$ and good desalination ability (Wang, K., et al., 2019). The experimental results reveal that it is an effective strategy to prepare Faradaic electrodes with good conductivity and high CDI performance derived from MOFs. To develop efficient, cheap, and safe Faradaic MOFC-based electrodes, more synthetic strategies of carbon materials combining MOFs with Faradaic materials need to be investigated.

As we discussed above, most MOFCs with high CDI performance usually involve controlled morphology, heteroatom doping, and Faradaic material doping. These modification methods are applied comprehensively in the synthesis of MOFCs with the purpose to optimize the nanostructure and composition of carbon materials, so as to achieve faster adsorption rate, higher SAC, and better cycling stability. The cases mentioned above with synthesis procedures and CDI performances are listed in Table $\mathbf{1 .}$ 
TABLE 1 | Typical cases of carbon electrodes derived from MOFs.

\begin{tabular}{|c|c|c|c|c|c|c|c|c|}
\hline Electrode & Precursor & Template & Heteroatom & $\begin{array}{l}\text { Faradaic } \\
\text { Material }\end{array}$ & Processing & $\begin{array}{l}\text { Electrochemical } \\
\text { Properties }\end{array}$ & $\begin{array}{l}\text { Desalination } \\
\text { Performance }\end{array}$ & $\begin{array}{l}\text { Cycling } \\
\text { Stability }\end{array}$ \\
\hline $\begin{array}{l}\text { IRMOF-1-derived } \\
\text { Carbon (Yang et al., } \\
\text { 2014) }\end{array}$ & IRMOF-1 & / & / & / & $\begin{array}{l}\text { Solvent evaporation } \\
\text { method; } 900^{\circ} \mathrm{C} \text {, } \\
\text { nitrogen }\end{array}$ & $\begin{array}{l}\sim 138 \mathrm{~F} \mathrm{~g}^{-1} \\
2 \mathrm{mV} \mathrm{s}^{-1}, 1 \mathrm{M} \\
\mathrm{NaCl}\end{array}$ & $\begin{array}{l}\sim 11 \mathrm{mg} \mathrm{g}^{-1}, 1.2 \mathrm{~V} \\
585 \mathrm{mg} \mathrm{L}^{-1}\end{array}$ & Not available \\
\hline $\begin{array}{l}\text { PCPs (Liu et al., } \\
\text { 2015b) }\end{array}$ & ZIF-8 & / & $\mathrm{N}$ & / & $\begin{array}{l}\text { Chemical reaction } \\
\text { at room } \\
\text { temperature; } \\
1,200^{\circ} \mathrm{C} \text {, nitrogen, } \\
\text { acid etching }\end{array}$ & $\begin{array}{l}275.69 \mathrm{~F} \mathrm{~g}^{-1} \\
1 \mathrm{mV} \mathrm{s}^{-1}, 1 \mathrm{M} \\
\mathrm{NaCl}\end{array}$ & $\begin{array}{l}13.86 \mathrm{mg} \mathrm{g}^{-1} \\
1.2 \mathrm{~V}, 500 \mathrm{mg} \mathrm{L}^{-1}\end{array}$ & $\begin{array}{l}\text { No obvious } \\
\text { electrosorption } \\
\text { capacity } \\
\text { declination after } \\
30 \text { cycles }\end{array}$ \\
\hline $\begin{array}{l}\text { Carbon Polyhedron } \\
\text { and carbon } \\
\text { Nanotube Hybrids } \\
\text { (Gao et al., 2018) }\end{array}$ & $\begin{array}{l}\text { ZIF- } \\
67 / \text { carbon } \\
\text { nanotubes }\end{array}$ & ZIF-67 & $\mathrm{N}$ & $\mathrm{Co}_{x} \mathrm{O}_{y}$ & $\begin{array}{l}\text { Chemical reaction } \\
\text { at } 40^{\circ} \mathrm{C} \text {; CVD } \\
\text { treatment }\end{array}$ & $\begin{array}{l}343 \mathrm{~F} \mathrm{~g}^{-1}, \\
10 \mathrm{mV} \mathrm{s}^{-1}, 6 \mathrm{M} \\
\mathrm{KOH}\end{array}$ & $\begin{array}{l}7.08 \mathrm{mg} \mathrm{g}^{-1}, 1.2 \mathrm{~V} \\
500 \mathrm{mg} \mathrm{L}^{-1}\end{array}$ & Not available \\
\hline $\begin{array}{l}\text { Shuttle-like porous } \\
\text { carbon rods (Xu } \\
\text { et al., 2016a) }\end{array}$ & MIL-88 (Fe) & / & / & / & $\begin{array}{l}\text { Hydrothermal } \\
\text { method; } 900^{\circ} \mathrm{C} \text {, } \\
\text { nitrogen; acid } \\
\text { etching }\end{array}$ & $\begin{array}{l}223.2 \mathrm{~F} \mathrm{~g}^{-1} \\
1 \mathrm{M} \mathrm{NaCl}\end{array}$ & $\begin{array}{l}16.2 \mathrm{mg} \mathrm{g}^{-1}, 1.2 \mathrm{~V} \\
1,000 \mathrm{mg} \mathrm{L}^{-1}\end{array}$ & $\begin{array}{l}95.1 \% \text { after } 30 \\
\text { cycles }\end{array}$ \\
\hline $\begin{array}{l}\text { NTP/C (Wang, K., } \\
\text { et al., 2019) }\end{array}$ & $\begin{array}{l}\mathrm{MIL}-125 \\
(\mathrm{Ti}) / \mathrm{NaH}_{2} \mathrm{PO}_{4}\end{array}$ & $\begin{array}{l}\text { MIL-125 } \\
\text { (Ti) derived } \\
\mathrm{TiO}_{2} / \text { carbon }\end{array}$ & / & $\mathrm{NaTi}_{2}\left(\mathrm{PO}_{4}\right)_{3}$ & $\begin{array}{l}\text { Solvothermal } \\
\text { method; } 600^{\circ} \mathrm{C} \text {, } \\
\text { nitrogen; } \\
\text { solvothermal, } \\
700^{\circ} \mathrm{C} \text {, nitrogen }\end{array}$ & $\begin{array}{l}164.8 \mathrm{~F} \mathrm{~g}^{-1} \\
10 \mathrm{mV} \mathrm{s}^{-1}, 1 \mathrm{M} \\
\mathrm{Na} 2 \mathrm{SO} 4\end{array}$ & $\begin{array}{l}167.4 \mathrm{mg} \mathrm{g}^{-1} \\
1.8 \mathrm{~V}, 3,000 \mathrm{mg} \mathrm{L}^{-1}\end{array}$ & $\begin{array}{l}90 \% \text { after } 30 \\
\text { cycles }\end{array}$ \\
\hline $\begin{array}{l}\text { Porous carbon } \\
\text { (Chang et al., 2015) }\end{array}$ & MOF-5 & / & / & / & $\begin{array}{l}\text { Chemical reaction } \\
\text { at } 85^{\circ} \mathrm{C} ; 900^{\circ} \mathrm{C} \text {, } \\
\text { vacuum }\end{array}$ & $\begin{array}{l}107.74 \mathrm{~F} \mathrm{~g}^{-1} \\
50 \mathrm{mV} \mathrm{s}^{-1} \\
0.5 \mathrm{M} \mathrm{NaCl}^{-}\end{array}$ & $\begin{array}{l}9.39 \mathrm{mg} \mathrm{g}^{-1}, 1.2 \mathrm{~V} \\
500 \mathrm{mg} \mathrm{L}^{-1}\end{array}$ & $\begin{array}{l}97.5 \% \text { after } 10 \\
\text { cycles }\end{array}$ \\
\hline $\begin{array}{l}\text { 3D-FeNC tubes (Xu } \\
\text { et al., 2020a) }\end{array}$ & $\begin{array}{l}\text { PAN@ZIF' } \\
\text { fiber }\end{array}$ & $\begin{array}{l}\mathrm{Zn} / \mathrm{PAN} \\
\text { fibers }\end{array}$ & $\mathrm{N}, \mathrm{Fe}$ & / & $\begin{array}{l}\text { Electrospinning } \\
\text { method, LBL } \\
\text { growth method, } \\
\text { template } \\
\text { dissolution; } 900^{\circ} \mathrm{C} \text {, } \\
\text { nitrogen }\end{array}$ & $\begin{array}{l}\mathrm{E}_{\text {onset }}: 0.98 \mathrm{~V} \\
\mathrm{E}_{1 / 2}: 0.877 \mathrm{~V} \\
10 \mathrm{mV} \mathrm{s}^{-1} \\
0.1 \mathrm{M} \mathrm{KOH}^{-}\end{array}$ & $\begin{array}{l}40.7 \mathrm{mg} \mathrm{g}^{-1}, 1.2 \mathrm{~V} \\
3,500 \mathrm{mg} \mathrm{L}^{-1}\end{array}$ & $\begin{array}{l}93.82 \% \text { after } \\
200 \text { cycles } \\
\text { (oxygenated } \\
\text { water) }\end{array}$ \\
\hline $\begin{array}{l}\text { hCNTs/PCP (Xu } \\
\text { et al., 2016b) }\end{array}$ & $\begin{array}{l}\text { CNTs/ZIF- } \\
8\end{array}$ & CNTS & $N$ & / & $\begin{array}{l}\text { in situ insertion of } \\
\text { CNTs in ZIF-8; } \\
1,000^{\circ} \mathrm{C} \text {, nitrogen }\end{array}$ & $\begin{array}{l}104.2 \mathrm{~F} \mathrm{~g}^{-1} \\
5 \mathrm{mV} \mathrm{s}^{-1}, 1 \mathrm{M} \\
\mathrm{NaCl}\end{array}$ & $\begin{array}{l}20.5 \mathrm{mg} \mathrm{g}^{-1}, 1.2 \mathrm{~V} \\
1,000 \mathrm{mg} \mathrm{L}^{-1}\end{array}$ & $\begin{array}{l}\text { No obvious } \\
\text { electrosorption } \\
\text { capacity } \\
\text { declination after } \\
30 \text { cycles }\end{array}$ \\
\hline $\begin{array}{l}\text { NCTs (Xu et al., } \\
2020 b)\end{array}$ & $\begin{array}{l}\text { PAN@ZIF- } \\
8\end{array}$ & $\mathrm{PAN} / \mathrm{Zn}(\mathrm{Ac})_{2}$ & $\mathrm{~N}$ & / & $\begin{array}{l}\text { Electrospinning, } \\
\text { LBL growth } \\
\text { method, template } \\
\text { dissolution; } 900^{\circ} \mathrm{C} \text {, } \\
\text { nitrogen }\end{array}$ & $\begin{array}{l}\sim 292 \mathrm{~F} \mathrm{~g}^{-1}, \\
10 \mathrm{mV} \mathrm{s}^{-1}, 1 \mathrm{M} \\
\mathrm{NaCl}\end{array}$ & $\begin{array}{l}56.9 \mathrm{mg} \mathrm{g}^{-1}, 1.2 \mathrm{~V} \\
3,500 \mathrm{mg} \mathrm{L}^{-1}\end{array}$ & $\begin{array}{l}96.9 \% \text { after } 50 \\
\text { cycles }\end{array}$ \\
\hline $\begin{array}{l}\text { NGCPs (Gao et al., } \\
\text { 2019) }\end{array}$ & ZIF-8 & / & $\mathrm{N}$ & / & $\begin{array}{l}\text { Chemical reaction } \\
\text { at room } \\
\text { temperature; } \\
1,000^{\circ} \mathrm{C} \text {, nitrogen } \\
\text { (low pressure) }\end{array}$ & $\begin{array}{l}307.4 \mathrm{~F} \mathrm{~g}^{-1}, \\
10 \mathrm{mV} \mathrm{s}^{-1}, 1 \mathrm{M} \\
\mathrm{NaCl}\end{array}$ & $\begin{array}{l}17.73 \mathrm{mg} \mathrm{g}^{-1} \\
1.4 \mathrm{~V}, 500 \mathrm{mg} \mathrm{L}^{-1}\end{array}$ & $\begin{array}{l}90.8 \% \text { after } 10 \\
\text { cycles }\end{array}$ \\
\hline $\begin{array}{l}\text { ZIF-8@PZS-C } \\
\text { (Zhang et al., 2018) }\end{array}$ & $\begin{array}{l}\text { ZIF- } \\
8 @ P Z S\end{array}$ & ZIF-8 & $N, P, S$ & I & $\begin{array}{l}\text { Electrostatic } \\
\text { interaction } \mathrm{PZS} \\
\text { coating; } 900^{\circ} \mathrm{C} \text {, } \\
\text { nitrogen, acid } \\
\text { etching }\end{array}$ & $\begin{array}{l}333 \mathrm{~F} \mathrm{~g}^{-1}, 1 \mathrm{mV} \\
\mathrm{s}^{-1}, 0.5 \mathrm{M} \mathrm{NaCl}\end{array}$ & $\begin{array}{l}22.19 \mathrm{mg} \mathrm{g}^{-1} \\
1.2 \mathrm{~V}, 500 \mathrm{mg} \mathrm{L}^{-1}\end{array}$ & $\begin{array}{l}99 \% \text { after } 20 \\
\text { cycles }\end{array}$ \\
\hline $\begin{array}{l}\text { HCZ (Yang and } \\
\text { Luo, 2019) }\end{array}$ & UiO-66 & / & / & $\mathrm{ZrO}_{2}$ & $\begin{array}{l}\text { Hydrothermal } \\
\text { method; } 900^{\circ} \mathrm{C} \text {, } \\
\text { nitrogen }\end{array}$ & $\begin{array}{l}128 \mathrm{~F} \mathrm{~g}^{-1}, 5 \mathrm{mV} \\
\mathrm{s}^{-1}, 1 \mathrm{M} \mathrm{NaCl}\end{array}$ & $\begin{array}{l}55.17 \mathrm{mg} \mathrm{g}^{-1} \\
1.4 \mathrm{~V}, 250 \mathrm{mg} \mathrm{L}^{-1}\end{array}$ & $\begin{array}{l}95.3 \% \text { after } 6 \\
\text { cycles }\end{array}$ \\
\hline $\begin{array}{l}\mathrm{TiO}_{2} @ \mathrm{PC}(\mathrm{Ding}, \mathrm{M} . \text {, } \\
\text { et al., 2019) }\end{array}$ & $\begin{array}{l}\text { MIL-125 } \\
\text { (Ti) }\end{array}$ & / & / & $\mathrm{TiO}_{2}$ & $\begin{array}{l}\text { Chemical reaction } \\
\text { at room } \\
\text { temperature; } \\
600^{\circ} \mathrm{C} \text {, argon }\end{array}$ & $\begin{array}{l}\sim 260 \mathrm{~F} \mathrm{~g}^{-1}, \\
10 \mathrm{mV} \mathrm{s}^{-1}, 1 \mathrm{M} \\
\mathrm{NaCl}\end{array}$ & $\begin{array}{l}46.7 \mathrm{mg} \mathrm{g}^{-1} \\
10 \mathrm{~mA} \mathrm{~g}^{-1} \\
1,000 \mathrm{mg} \mathrm{L}^{-1}\end{array}$ & $\begin{array}{l}\text { No obvious } \\
\text { electrosorption } \\
\text { capacity } \\
\text { declination after } \\
54 \text { cycles }\end{array}$ \\
\hline
\end{tabular}




\section{CONCLUSIONS AND OUTLOOK}

As a potential water treatment technology, $\mathrm{CDI}$ is progressively making its path to the desalination industry. In this process, the first and most important is the development of high-efficiency and low-cost electrode materials. Nanocarbon materials derived from metal-organic frameworks have become one of the most promising candidates for their highly designable precursors. Thanks to the application of creative modification methods, breakthroughs have been made in the CDI performance of MOFCs.

Nevertheless, promotion of desalination efficiency is merely the first step of industrialization, the next will be the control of cost. Generally, the synthesis of MOFCs should select a wide range of cheap raw materials and simple synthetic routes. For example, MILs composed of metal ions such as iron, titanium, manganese, and organic ligands such as fumaric acid and terephthalic acid may be an ideal choice due to their low cost, safety, and high specific surface area. In terms of morphology control, other than the template strategies mentioned above, more methods need to be investigated. Nitrogen doping is a common modification method of MOFCs with a main consideration of nitrogen source. In addition to nitrogencontaining MOFs, cheap external nitrogen sources such as urea and ammonia are also worth considering. In the aspect of MOFCbased Faradaic electrode, transition metal oxides (Kai et al., 2017) and polyanionic phosphates with low price and high salt adsorption ability and are environmentally friendly hold great potential.

In summary, MOFCs are one of the most promising electrode materials for CDI. The further developing target is to achieve

\section{REFERENCES}

AlMarzooqi, F. A., Al Ghaferi, A. A., Saadat, I., and Hilal, N. (2014). Application of capacitive deionisation in water desalination: a review. Desalination 342, 3-15. doi: 10.1016/j.desal.2014.02.031

Chaikittisilp, W., Ariga, K., and Yamauchi, Y. (2013). A new family of carbon materials: synthesis of MOF-derived nanoporous carbons and their promising applications. J. Mater. Chem. A 1, 14-19. doi: 10.1039/C2TA0 0278G

Chang, L., Li, J., Duan, X., and Liu, W. (2015). Porous carbon derived from Metalorganic framework (MOF) for capacitive deionization electrode. Electrochim. Acta 176, 956-964. doi: 10.1016/j.electacta.2015.07.130

Chen, Z., Xu, X., Ding, Z., Wang, K., Sun, X., Lu, T., et al. (2020). Ti3C2 MXenes-derived NaTi2(PO4)3/MXene nanohybrid for fast and efficient hybrid capacitive deionization performance. Chem. Eng. J. 2020:127148. doi: 10.1016/j.cej.2020.127148

Cheng, Y., Hao, Z., Hao, C., Deng, Y., Li, X., Li, K., et al. (2019). A review of modification of carbon electrode material in capacitive deionization. RSC Adv. 9, 24401-24419. doi: 10.1039/C9RA04426D

Dang, S., Zhu, Q.-L., and Xu, Q. (2017). Nanomaterials derived from metalorganic frameworks. Nat. Rev. Mater. 3:17075. doi: 10.1038/natrevmats.2017.75

Ding, M., Fan, S., Huang, S., Pam, M. E., Guo, L., Shi, Y., et al. (2019), Tunable pseudocapacitive behavior in metal-organic framework-derived $\mathrm{TiO}_{2} @$ porous carbon enabling high-performance membrane capacitive deionization. ACS Appl. Energy Mater. 2, 1812-1822. doi: 10.1021/acsaem.8b 01839 higher SAC, faster desalination rate, higher cycling stability, environmental friendliness, and lower cost. Considering that recent studies have revealed the outstanding performance of hybrid CDI with Faradaic negative electrodes, Faradaic material doping might become a mainstream modification method. Moreover, since the current CDI positive electrode materials are still carbon materials, it is vital to improve the non-Faraday desalination performance of MOFCs through morphology control and element doping. It can be expected that the combination of Faradaic mechanism and nonFaradaic mechanism by selecting appropriate modification methods of MOFCs would give CDI better desalination performance.

\section{AUTHOR CONTRIBUTIONS}

PL, ML, XS, and YW: proposal and writing. TY and XX: revising and guidance. All authors contributed to the article and approved the submitted version.

\section{FUNDING}

This work was supported by the Fundamental Research Funds for the Central Universities (B200202034) and National Natural Science Foundation of China (51909066).

\section{SUPPLEMENTARY MATERIAL}

The Supplementary Material for this article can be found online at: https://www.frontiersin.org/articles/10.3389/fchem. 2020.575350/full\#supplementary-material

Ding, Z., Xu, X., Li, Y., Wang, K., Lu, T., and Pan, L. (2019). Significantly improved stability of hybrid capacitive deionization using nickel hexacyanoferrate/reduced graphene oxide cathode at low voltage operation. Desalination 468:114078. doi: 10.1016/j.desal.2019.114078

Duan, F., Du, X., Li, Y., Cao, H., and Zhang, Y. (2015). Desalination stability of capacitive deionization using ordered mesoporous carbon: effect of oxygencontaining surface groups and pore properties. Desalination 376, 17-24. doi: 10.1016/j.desal.2015.08.009

Dutta, S., Huang, S.-Y., Chen, C., Chen, J. E., Alothman, Z. A., Yamauchi, Y., et al. (2016). Cellulose framework directed construction of hierarchically porous carbons offering high-performance capacitive deionization of brackish water. ACS Sust. Chem. Eng. 4, 1885-1893. doi: 10.1021/acssuschemeng.5b01587

Gao, T., Du, Y., and Li, H. (2019). Preparation of nitrogen-doped graphitic porous carbon towards capacitive deionization with high adsorption capacity and rate capability. Separat. Purif. Technol. 211, 233-241. doi: 10.1016/j.seppur.2018.09.085

Gao, T., Zhou, F., Ma, W., and Li, H. (2018). Metal-organic-framework derived carbon polyhedron and carbon nanotube hybrids as electrode for electrochemical supercapacitor and capacitive deionization. Electrochim. Acta 263, 85-93. doi: 10.1016/j.electacta.2018.01.044

Hou, S., Xu, X., Wang, M., Lu, T., Sun, C. Q., and Pan, L. (2018). Synergistic conversion and removal of total $\mathrm{Cr}$ from aqueous solution by photocatalysis and capacitive deionization. Chem. Eng. J. 398-404. doi: 10.1016/j.cej.2017.12.120

Huang, H., Yan, M., Yang, C., He, H., Jiang, Q., Yang, L., et al. (2019). Graphene nanoarchitectonics: recent advances in graphene-based 
electrocatalysts for hydrogen evolution reaction. Adv. Mater. 31:1903415. doi: 10.1002/adma.201903415

Jung, H.-H., Hwang, S.-W., Hyun, S.-H., Lee, K.-H., and Kim, G.-T. (2007). Capacitive deionization characteristics of nanostructured carbon aerogel electrodes synthesized via ambient drying. Desalination 216, 377-385. doi: 10.1016/j.desal.2006.11.023

Kai, W., Liwei, L., Wen, X., Shengzhe, Z., Yong, L., Hongwei, Z., et al. (2017). Electrodeposition synthesis of $\mathrm{PANI} / \mathrm{MnO}_{2} /$ graphene composite materials and its electrochemical performance. Int. J. Electrochem. Sci. 12, 8306-8314. doi: 10.20964/2017.09.06

Kurak, K. A., and Anderson, A. B. (2009). Nitrogen-treated graphite and oxygen electroreduction on pyridinic edge sites. J. Phys. Chem. C 113, 6730-6734. doi: $10.1021 /$ jp811518e

Li, H., Pan, L., Nie, C., Liu, Y., and Sun, Z. (2012). Reduced graphene oxide and activated carbon composites for capacitive deionization. J. Mater. Chem. 22, 15556-15561. doi: 10.1039/c2jm32207b

Li, Y., Liu, Y., Wang, M., Xu, X., Lu, T., Sun, C., et al. (2018). Phosphorusdoped 3D carbon nanofiber aerogels derived from bacterial-cellulose for highly-efficient capacitive deionization. Carbon. 130, 377-383. doi: 10.1016/j.carbon.2018.01.035

Liu, P., Yan, T., Shi, L., Park, H. S., Chen, X., Zhao, Z., et al. (2017). Graphenebased materials for capacitive deionization. J. Mater. Chem. A 5, 13907-13943. doi: 10.1039/C7TA02653F

Liu, X., Feng, G., Wu, Z., Yang, Z., Yang, S., Guo, X., et al. (2020). Enhanced sodium storage property of sodium vanadium phosphate via simultaneous carbon coating and Nb5+ doping. Chem. Eng. J. 386:123953. doi: 10.1016/j.cej.2019.123953

Liu, Y., Nie, C., Liu, X., Xu, X., Sun, Z., and Pan, L. (2015a). Review on carbon-based composite materials for capacitive deionization. RSC Adv. 5, 15205-15225. doi: 10.1039/C4RA14447C

Liu, Y., Xu, X., Wang, M., Lu, T., Sun, Z., and Pan, L. (2015b). Metal-organic framework-derived porous carbon polyhedra for highly efficient capacitive deionization. Chem. Commun. 51, 12020-12023. doi: 10.1039/C5CC0 3999A

Lu, T., Liu, Y. Xu, X., Pan, L., Alothman, A. A., Shapter, J., et al. (2020). Highly efficient water desalination by capacitive deionization on biomassderived porous carbon nanoflakes. Separat. Purif. Technol. 256:117771. doi: 10.1016/j.seppur.2020.117771

Luo, J., Tian, D., Ding, Z., Lu, T., Xu, X., and Pan, L. (2019). Enhanced cycling stability of capacitive deionization via effectively inhibiting $\mathrm{H}_{2} \mathrm{O}_{2}$ formation: the role of nitrogen dopants. J. Electr. Chem. 855:113488. doi: 10.1016/j.jelechem.2019.113488

Oren, Y. (2008). Capacitive deionization (CDI) for desalination and water treatment - past, present and future (a review). Desalination 228, 10-29. doi: 10.1016/j.desal.2007.08.005

Qin, W., Li, J., Liu, X., Zhou, N., Wu, C., Ding, M., et al. (2019). Formation of needle-like porous $\mathrm{CoNi}_{2} \mathrm{~S}_{4}-\mathrm{MnOOH}$ for high performance hybrid supercapacitors with high energy density. J. Coll Interf. Sci. 554, 125-132. doi: $10.1016 /$ j.jcis.2019.07.010

Qin, W., Zhou, N., Wu, C., Xie, M., Sun, H., Guo, Y., et al. (2020). Minireview on the redox additives in aqueous electrolyte for high performance supercapacitors. ACS Omega 5, 3801-3808. doi: 10.1021/acsomega.9b04063

Sun, X., Jia, X., Liu, J., Wang, G., Zhao, S., Ji, L., et al. (2020a). Investigation on the characteristics of an advanced rotational hydrodynamic cavitation reactor for water treatment. Separat. Purif. Technol. 251:117252. doi: 10.1016/j.seppur.2020.117252

Sun, X., Liu, J., Ji, L., Wang, G., Zhao, S., Yoon, J. Y., et al. (2020b). A review on hydrodynamic cavitation disinfection: the current state of knowledge. Sci. Total Environ. 737:139606. doi: 10.1016/j.scitotenv.2020. 139606

Suss, M., Porada, S., Sun, X., Biesheuvel, P., Yoon, J., and Presser, V. (2015). Water desalination via capacitive deionization: what is it and what can we expect from it? Energy Environ. Sci. 8, 2296-2319. doi: 10.1039/C5EE00519A

Tang, H., Cai, S., Xie, S., Wang, Z., Tong, Y., Pan, M., et al. (2016). Metalorganic-framework-derived dual metal-and nitrogen-doped carbon as efficient and robust oxygen reduction reaction catalysts for microbial fuel cells. Adv. Sci. 3:1500265. doi: 10.1002/advs.201500265
Tang, K., Hong, T. Z. X., You, L., and Zhou, K. (2019). Carbon-metal compound composite electrodes for capacitive deionization: synthesis, development and applications. J. Mate. Chem. A 7, 26693-26743. doi: 10.1039/C9TA08663C

Tang, W., Liang, J., He, D., Gong, J., Tang, L., Liu, Z., et al. (2019). Various cell architectures of capacitive deionization: recent advances and future trends. Water Res. 150, 225-251. doi: 10.1016/j.watres.2018.11.064

Wang, G., Dong, Q., Ling, Z., Pan, C., Yu, C., and Qiu, J. (2012). Hierarchical activated carbon nanofiber webs with tuned structure fabricated by electrospinning for capacitive deionization. J. Mater. Chem. 22, 21819-21823. doi: $10.1039 / \mathrm{c} 2 \mathrm{jm} 34890 \mathrm{j}$

Wang, G., Qian, B., Dong, Q., Yang, J., Zhao, Z., and Qiu, J. (2013). Highly mesoporous activated carbon electrode for capacitive deionization. Separat. Purif. Technol. 103, 216-221. doi: 10.1016/j.seppur.2012.10.041

Wang, K., Li, L., Zhang, T., and Liu, Z. (2014). Nitrogen-doped graphene for supercapacitor with long-term electrochemical stability. Energy 70, 612-617. doi: 10.1016/j.energy.2014.04.034

Wang, K., Liu, Y., Ding, Z., Li, Y., Lu, T., and Pan, L. (2019). Metalorganic-frameworks-derived $\mathrm{NaTi}_{2} \quad\left(\mathrm{PO}_{4}\right)$ 3/carbon composites for efficient hybrid capacitive deionization. J. Mater. Chem. A 7, 12126-12133. doi: 10.1039/C9TA01106D

Wang, L., Wang, M., Huang, Z.-H., Cui, T., Gui, X., Kang, F., et al. (2011). Capacitive deionization of $\mathrm{NaCl}$ solutions using carbon nanotube sponge electrodes. J. Mater. Chem. 21, 18295-18299. doi: 10.1039/c1jm13105b

Wang, M., Xu, X., Tang, J., Hou, S., Hossain, M. S. A., Pan, L., et al. (2017). High performance capacitive deionization electrodes based on ultrathin nitrogendoped carbon/graphene nano-sandwiches. Chem. Commun. 53, 10784-10787. doi: 10.1039/C7CC05673G

Wang, Z., Xu, X., Kim, J., Malgras, V., Mo, R., Li, C., et al. (2019). Nanoarchitectured metal-organic framework/polypyrrole hybrids for brackish water desalination using capacitive deionization. Mater. Horiz. 6, 1433-1437. doi: 10.1039/C9MH00306A

Xu, X., Allah, A. E., Wang, C., Tan, H., Farghali, A. A., Khedr, M. H., et al. (2019a). Capacitive deionization using nitrogen-doped mesostructured carbons for highly efficient brackish water desalination. Chem. Eng. J. 362, 887-896. doi: 10.1016/j.cej.2019.01.098

Xu, X., Li, C., Wang, C., Ji, L., Kaneti, Y. V., Huang, H., et al. (2019b). Threedimensional nanoarchitecture of carbon nanotube-interwoven metal-organic frameworks for capacitive deionization of saline water. ACS Sust. Chem. Eng. 7, 13949-13954. doi: 10.1021/acssuschemeng.9b02367

Xu, X., Li, J., Wang, M., Liu, Y., Lu, T., and Pan, L. (2016a). Shuttlelike porous carbon rods from carbonized metal-organic frameworks for high-performance capacitive deionization. ChemElectroChem 3, 993-998. doi: 10.1002/celc.201600051

Xu, X., Liu, Y., Wang, M., Zhu, C., Lu, T., Zhao, R., et al. (2016b). Hierarchical hybrids with microporous carbon spheres decorated three-dimensional graphene frameworks for capacitive applications in supercapacitor and deionization. Electrochim. Acta 193, 88-95. doi: 10.1016/j.electacta.2016.02.049

Xu, X., Pan, L., Liu, Y., Lu, T., and Sun, Z. (2015). Enhanced capacitive deionization performance of graphene by nitrogen doping. J. Coll Interf. Sci. 445, 143-150. doi: 10.1016/j.jcis.2015.01.003

Xu, X., Tan, H., Wang, Z., Wang, C., Pan, L., Kaneti, Y. V., et al. (2019c). Extraordinary capacitive deionization performance of highly-ordered mesoporous carbon nano-polyhedra for brackish water desalination. Environ. Sci. Nano 6, 981-989. doi: 10.1039/C9EN00017H

Xu, X., Tang, H., Wang, M., Liu, Y., Li, Y., Lu, T., et al. (2016c). Carbon spheres with hierarchical micro/mesopores for water desalination by capacitive deionization. J. Mater. Chem. A 4, 16094-16100. doi: 10.1039/C6TA07616E

Xu, X., Tang, J., Kaneti, Y. V., Tan, H., Chen, T., Pan, L., et al. (2020a). Unprecedented capacitive deionization performance of interconnected ironnitrogen-doped carbon tubes in oxygenated saline water. Mater. Horiz. 7, 1404-1412. doi: 10.1039/C9MH01829H

Xu, X., Tang, J., Qian, H., Hou, S., Bando, Y., Hossain, M. S. A., et al. (2017a). Three-dimensional networked metal-organic frameworks with conductive polypyrrole tubes for flexible supercapacitors. ACS Appl. Mater. Interf. 9, 38737-38744. doi: 10.1021/acsami.7b09944

Xu, X., Wang, M., Liu, Y., Lu, T., and Pan, L. (2016d). Metal-organic frameworkengaged formation of a hierarchical hybrid with carbon nanotube inserted 
porous carbon polyhedra for highly efficient capacitive deionization. J. Mater. Chem. A 4, 5467-5473. doi: 10.1039/C6TA00618C

Xu, X., Wang, M., Liu, Y., Lu, T., and Pan, L. (2017b). Ultrahigh desalinization performance of asymmetric flow-electrode capacitive deionization device with an improved operation voltage of 1.8 V. ACS Sust. Chem. Eng. 5, 189-195. doi: 10.1021/acssuschemeng.6b01212

Xu, X., Yang, T., Zhang, Q., Xia, W., Ding, Z., Eid, K., et al. (2020b). Ultrahigh capacitive deionization performance by $3 \mathrm{D}$ interconnected MOF-derived nitrogen-doped carbon tubes. Chem. Eng. J. 390:124493. doi: $10.1016 /$ j.cej.2020.124493

Yaghi, O., and Li, H. (1995). Hydrothermal synthesis of a metal-organic framework containing large rectangular channels. J. Am. Chem. Soc. 117, 10401-10402. doi: $10.1021 /$ ja00146a033

Yang, S., and Luo, M. (2019). In-situ embedding $\mathrm{ZrO}_{2}$ nanoparticles in hierarchically porous carbon matrix as electrode materials for high desalination capacity of hybrid capacitive deionization. Mater. Lett. 248, 197-200. doi: 10.1016/j.matlet.2019.04.019

Yang, S. J., Kim, T., Lee, K., Kim, Y. S., Yoon, J., and Park, C. R. (2014). Solvent evaporation mediated preparation of hierarchically porous metal organic framework-derived carbon with controllable and accessible large-scale porosity. Carbon 71, 294-302. doi: 10.1016/j.carbon.2014.01.056

Yin, H., Zhao, S., Wan, J., Tang, H., Chang, L., He, L., et al. (2013). Three-dimensional graphene/metal oxide nanoparticle hybrids for highperformance capacitive deionization of saline water. Adv. Mater. 25, 6270-6276. doi: 10.1002/adma.201302223

Yu, F., Wang, L., Wang, Y., Shen, X., Cheng, Y., and Ma, J. (2019). Faradaic reactions in capacitive deionization for desalination and ion separation. $J$. Mater. Chem. A 7, 15999-16027. doi: 10.1039/C9TA01264H
Zhang, J., Fang, J., Han, J., Yan, T., Shi, L., and Zhang, D. (2018). N, P, $S$ co-doped hollow carbon polyhedra derived from MOF-based core-shell nanocomposites for capacitive deionization. J. Mater. Chem. A 6, 15245-15252. doi: 10.1039/C8TA04813D

Zhang, S., Xia, W., Yang, Q., Kaneti, Y. V., Xu, X., Alshehri, S. M., et al. (2020). Core-shell motif construction: highly graphitic nitrogen-doped porous carbon electrocatalysts using MOF-derived carbon@ COF heterostructures as sacrificial templates. Chem. Eng. J. 396:125154. doi: 10.1016/j.cej.2020.1 25154

Zhao, W., Ding, M., Guo, L., and Yang, H. Y. (2019). Dual-ion electrochemical deionization system with binder-free aerogel electrodes. Small 15:1805505. doi: 10.1002/smll.201805505

Zheng, Y., Jiao, Y., Chen, J., Liu, J., Liang, J., Du, A., et al. (2011). Nanoporous graphitic-C3N4@ carbon metal-free electrocatalysts for highly efficient oxygen reduction. J. Am. Chem. Soc. 133, 20116-20119. doi: 10.1021/ ja209206c

Conflict of Interest: The authors declare that the research was conducted in the absence of any commercial or financial relationships that could be construed as a potential conflict of interest.

Copyright (๑) 2020 Lin, Liao, Yang, Sheng, Wu and Xu. This is an open-access article distributed under the terms of the Creative Commons Attribution License (CC BY). The use, distribution or reproduction in other forums is permitted, provided the original author(s) and the copyright owner(s) are credited and that the original publication in this journal is cited, in accordance with accepted academic practice. No use, distribution or reproduction is permitted which does not comply with these terms. 